\title{
Optimal Voltage and Reactive Power Scheduling for Saving Electric Charges using Dynamic Programming with a Heuristic Search Approach
}

\author{
Ki-Seok Jeong ${ }^{\dagger}$ and Jong-Duk Chung*
}

\begin{abstract}
With the increasing deployment of distributed generators in the distribution system, a very large search space is required when dynamic programming (DP) is applied for the optimized dispatch schedules of voltage and reactive power controllers such as on-load tap changers, distributed generators, and shunt capacitors. This study proposes a new optimal voltage and reactive power scheduling method based on dynamic programming with a heuristic searching space reduction approach to reduce the computational burden. This algorithm is designed to determine optimum dispatch schedules based on power system day-ahead scheduling, with new control objectives that consider the reduction of active power losses and maintain the receiving power factor.

In this work, to reduce the computational burden, an advanced voltage sensitivity index (AVSI) is adopted to reduce the number of load-flow calculations by estimating bus voltages. Moreover, the accumulated switching operation number up to the current stage is applied prior to the load-flow calculation module. The computational burden can be greatly reduced by using dynamic programming. Case studies were conducted using the IEEE 30-bus test systems and the simulation results indicate that the proposed method is more effective in terms of saving electric charges and improving the voltage profile than loss minimization.
\end{abstract}

Keywords: Optimal voltage and reactive power control, Dynamic programming, Voltage sensitivity index, Receiving power factor, Distributed generator

\section{Introduction}

Voltage control and reactive power compensation are effective means for reducing power and energy losses and improving energy quality in a power system. Thus, the problem arising due to an inadequate reactive power supply can be solved by reallocating reactive power generation in the system by adjusting the transformer taps, changing the generator voltages, and switching the VAR sources. To achieve this, it is important to develop algorithms that provide decision making to the set point of voltage controllers and reactive power sources using appropriate mathematical programming methods [1-2].

Research in the field of voltage and reactive power controls for distribution systems can be divided into two categories: offline setting control and real-time control [3]. Such power control methods can be applied to smart grids, formed by the integration of future power systems with IT technology using high-efficiency devices and systems [4]. Nowadays, as developing low-carbon generation technologies gain traction owing to the drive towards a green policy, different types of renewable energy systems are deployed in several microgrids.

The introduction of distributed generators (DGs) based

$\dagger$ Corresponding Author: Metropolitan Transit Convergence Research Division, Korea Railroad Research Institute, Korea. (jks14@krri.re.kr)

* Metropolitan Transit Convergence Research Division, Korea Railroad Research Institute, Korea. (jdchung@krri.re.kr)

Received: August 21, 2014; Accepted: November 18, 2015 on renewable energy resources has an impact on the voltage control and reactive power dispatch strategy [5]. Therefore, distribution network operators (DNOs) should find the optimal way for power system reliability, loss minimization, and the voltage profile improvement while providing energy supply services as the primary goal [6].

The general optimal reactive power dispatch problem (ORPD) is a very complicated, discrete and non-differentiable formulation with non-linear multi-objective function [7]. The conventional methods based on mathematical approaches have main disadvantage due to trapping in a local minima. To overcome traditional method, various heuristic methods based on deterministic and stochastic approaches have been introduced. Ying-Tung Hsiao et al. [8] applied the simulated annealing (SA) to optimal multiobjective VAR planning in large scale power system. Z. Hu et al. [3] applied the genetic algorithm (GA) to voltage and VAR control in distribution systems using time-interval based approach. Wanik, M.Z.C. et al. [4] applied the particle swarm optimization (PSO) to distributed generators reactive power management in distribution network. Wen Zhang et al. [7] and M.E. Hamedani Golshan et al. [9] proposed the solution of optimal var planning using improved Tabu Search (TS) techniques.

Dynamic programming is one of the deterministic approaches of heuristic optimization techniques and can be used to overcome the disadvantage of existing analytical optimization methods such as LP and NLP. However, when 
DP is employed to decide the optimal schedules of several equipment, the computational load is acceptable due to the relatively little state space [10]. Nevertheless, to determine the optimal scheduling of more controllers, huge search space is required, and this is computationally timeconsuming [11-12]. Novel techniques should be applied to the algorithm to reduce the computational burden. If a reduced computational load ensures a feasible searching space using an optimization method such as proposed approach, it could be one of the best solution engines for addressing the ORPD problem. Proposed approach can provide decision making capability available to the set point of the voltage controllers and reactive power sources based on day-ahead hourly power system profile.

This study deal with voltage and reactive power coordination algorithm to solve the off-line setting control problem by coordinating all voltage and reactive power control devices in a distribution system with DGs. The aim of this paper is to find the optimal dispatch schedule for all such devices in the distribution system so that the total electric charges can be minimized. The proposed objective function considers system active losses and receiving power factor.

\section{Problem Formulation}

A coordinated control strategy between on-load tap changers (OLTCs), DG units, and shunt capacitors in the distribution power system can be considered as a nonlinear discrete optimization problem. The control objective function of this problem is to minimize the electric charges, by considering active power losses and the receiving power factor at the point of common coupling (PCC) based on the forecasted hourly loads of each bus [13]. With the hourly power system day-ahead scheduling, the proposed method aims to find dispatch schedules for positions and DG setting and status of all shunt capacitors according to an optimization process based on the load demand and the DG output for the day ahead [11]. The optimal dispatch schedules for setting OLTCs and DG units and for switching shunt capacitors could be formulated mathematically [7]. The control objective in Eq. (1) is set to minimize the electric charges, by including real power losses and the receiving power factor at time $i$ and maintain all nodal voltages within the desired limits [9]:

$$
\text { Min. } \mathbf{J}=\sum_{\mathbf{i}=\mathbf{1}}^{\mathbf{N}}\left[\mathbf{C}_{\text {Ploss }} \cdot \mathbf{P}_{\text {Loss }, \mathbf{i}}+\mathbf{C}_{\mathbf{P F}, \mathbf{i}} \cdot\left(\mathbf{P F}_{\mathbf{i}}^{\text {spec }}-\mathbf{P F}_{\mathbf{i}}\right)\right]
$$

The objective function in Eq. (1) is subject to the standard power balancing equality constraints and to the following additional inequality constraints:

$$
V_{i, k}^{\min } \leq V_{i, k} \leq V_{i, k}^{\max }
$$

$$
\begin{gathered}
T^{\min } \leq T^{\prime} \leq T_{\max } \\
C_{\mathbf{w}}^{\min } \leq \mathbf{C}_{\mathbf{w}} \leq \mathbf{C}_{\mathbf{w}}^{\max }
\end{gathered}
$$

The daily switching operation number (SON) of each control variable is applied as a coupling constraint with the maximum SON.

$$
\begin{aligned}
& \mathbf{N K}_{\text {tap }}=\sum_{\mathbf{i}=1}^{\mathbf{N}}\left|\mathbf{T A P}_{\mathbf{i}}-\mathbf{T A P} \mathbf{P}_{\mathbf{i}-\mathbf{1}}\right| \leq \mathbf{M K} \mathbf{T}_{\mathbf{N}} \\
& \mathrm{NK}_{\mathrm{C}, \mathrm{w}}=\sum_{\mathrm{i}=\mathbf{1}}^{\mathrm{N}}\left|\mathrm{C}_{\mathrm{w}, \mathrm{i}}-\mathrm{C}_{\mathrm{w}, \mathrm{i}-\mathbf{1}}\right| \leq \mathrm{MK}_{\mathrm{C}, \mathrm{w}} \\
& \mathbf{N K}_{\mathbf{G}}=\sum_{\mathbf{i}=\mathbf{1}}^{\mathbf{N}}\left|\mathbf{V G}_{\mathbf{i}}-\mathbf{V G}_{\mathbf{i}-\mathbf{1}}\right| \leq \mathrm{MK}_{\mathbf{G}}
\end{aligned}
$$

In Eqs. (1) - (7), the additional variables are defined as follows:

$\mathbf{N}$ number of stages in a day, which is 24 for onehour intervals between $i$ and $i+1$

C $_{\text {Ploss }}$ cost-weighting factor for active power loss

$\mathbf{P}_{\text {Loss, i }}$ total active power loss at time $i$

$\mathbf{C}_{\mathbf{P F}, \mathbf{i}} \quad$ cost-weighting factor for power factor at time $i$

$\mathbf{P F}_{\mathbf{i}}^{\text {spec }} \quad$ specific power factor at time $i$, i.e., 0.9 p.u.

$\mathbf{P F}_{\mathbf{i}} \quad$ receiving power factor at time $i$

$\mathbf{V}_{\mathbf{i}, \mathbf{k}}^{\mathbf{m i n}} \quad$ minimum permissible voltage, i.e., 0.97 p.u.

$\mathbf{V}_{\mathbf{i}, \mathbf{k}} \quad$ maximum permissible voltage, i.e., 1.03 p.u.

$\mathbf{T}^{\mathbf{m i n}} \quad$ lower bound of tap position, i.e., -8

$\mathbf{T}^{\mathbf{m a x}} \quad$ upper bound of tap position, i.e., +8

$\mathbf{C}_{\mathbf{w}}^{\min } \quad$ lower bound of $\mathrm{w}$-th shunt capacitor, i.e., 0

$\mathbf{C}_{\mathbf{w}}^{\max } \quad$ upper bound of $\mathrm{w}$-th shunt capacitor, i.e., 1

$\mathbf{N K}_{\text {tap }}, \mathbf{N K}_{\mathbf{G}}$ SON of OLTCs and DGs

$\mathbf{M K}_{\text {tap }}, \mathbf{M K}_{\mathbf{G}}$ maximum SON of OLTCs and DGs

The control objective of Eq. (1) is divided into two parts as described in Fig. 1 in [13]. Fig. 1 represents two costweighting coefficients: usage cost for active power losses (a solid black line) and daytime and night-time demand charge (dotted blue line and dot-and-dash red line). The gradient of the usage cost line is $65 \mathrm{KRW} / \mathrm{KWh}$. In the daytime, if the receiving power factor with the lagging mode is maintained between $90 \%$ and $95 \%$, there is

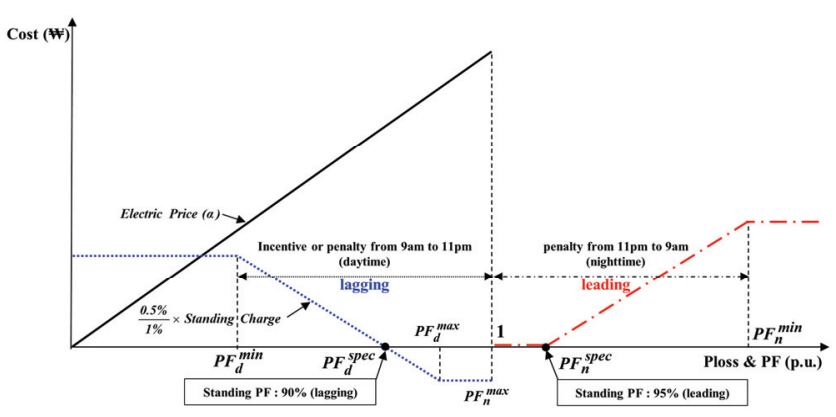

Fig. 1. Cost-weighting factors of objective function in [7] 
economic incentive; if the receiving power factor is not maintained above $90 \%$, there is penalty. There is only penalty in the night-time, if the receiving power factor with a leading operation mode is maintained above $95 \%$.

The first sub-objective function in Eq. (1) is the usage cost per hour multiplied active power losses by electricity price. To reflect the actual billing system of KEPCO, the electric charges in an electric service agreement are assumed as the electricity price per unit, $\mathrm{C}_{\text {Ploss }}$, which is 65 $\mathrm{KRW} / \mathrm{kWh}$ [14]. The second part of Eq. (1) is the demand charge of the receiving power factor penalty or incentive, which is the product of the standing charge and the power factor rate [13], as given by the following equation:

$$
\mathrm{C}_{\mathrm{PF}, \mathrm{i}}=\mathrm{C}_{\mathrm{SC}} \cdot \boldsymbol{\lambda}_{\mathrm{i}}
$$

where $\mathrm{C}_{\mathrm{SC}}$ is the standing charge per hour, 106,944 KRW/h based on $11 \mathrm{MW}$ contract power from KEPCO in this study. The value $\lambda_{\mathrm{i}}$ is the penalty or incentive factor of the power factor charge applied to the electric service agreement as given by the following equation:

$$
\lambda_{i, d}=\left\{\begin{array}{c}
+15, P F<P F_{d}^{\text {min }} \\
0.5 \cdot\left(P_{d}^{\text {spec }}-\mathrm{PF}\right), \mathrm{PF}_{\mathrm{d}}^{\text {min }} \leq \mathrm{PF} \leq \mathrm{PF}_{\mathrm{d}}^{\mathrm{spec}} \\
0.5 \cdot\left(\mathrm{PF}_{\mathrm{d}}^{\mathrm{spec}}-\mathrm{PF}\right), \mathrm{PF}_{\mathrm{d}}^{\mathrm{spec}} \leq \mathrm{PF} \leq \mathrm{PF}_{\mathrm{d}}^{\max } \\
-2.5, \mathrm{PF}_{\mathrm{d}}^{\text {max }} \leq \mathrm{PF}<1
\end{array}\right\}
$$

where $\lambda_{\mathrm{i}, \mathrm{d}}$ is the incentive or penalty factor of the receiving power factor in daytime $(9 \leq i \leq 24), \mathrm{PF}_{\mathrm{d}}^{\mathrm{spec}}$ is the standing power factor in daytime, i.e., $90 \%$ lagging, $\mathrm{PF}_{\mathrm{d}}^{\min }$ is the minimum power factor, which has a maximum penalty in daytime, i.e., $60 \%$, and $\mathrm{PF}_{\mathrm{d}}^{\max }$ is the maximum power factor, which has a maximum incentive in daytime, i.e., $95 \%$.

\section{Solution Algorithm}

The optimal dispatch problem is mathematically formulated in the previous section. In this section, the solution methodology to determine an optimal dispatch with proposed approach based on VSI is discussed. The solution engine with the proposed method is developed to automatically run the load flow, calculate the VSI and system losses, and ultimately search for the optimum path in a PSS/E environment with python [15].

\subsection{Advanced voltage sensitivity index (AVSI)}

The VSI could be adopted for minimizing the computational effort mainly depending on iterative load flow calculation. The conventional VSI at each bus is obtained from the inverse matrix of the reduced jacobian matrix, which directly relates the node voltage magnitude to reactive power injection as the following equation [16-18]:

$$
\mathrm{VSI}_{\mathbf{k}}=\Delta \mathbf{V}_{\mathbf{k}} / \Delta \mathbf{Q}_{\mathbf{k}}
$$

This index is row vector consists of some components represented as control variables. Each component is represented as VSI of shunt capacitors, OLTCs and DG units. The conventional VSI is obtained from the matrix consisting of voltage deviation factors (VDFs) obtained from two load flow calculation of initial state and state after one-step changed. Estimated bus voltage at each bus can be obtained from sum of initial bus voltage and voltage deviation in Eq. (11).

$$
\mathbf{V}_{\mathbf{k}}^{\text {est }}=\mathbf{V}_{\mathbf{k}}^{\text {ini }}+\Delta \mathbf{V}_{\mathbf{k}} \quad \mathbf{k} \in \mathbf{N}_{\mathbf{B}}
$$

where $\mathrm{V}_{\mathrm{k}}^{\text {est }}$ is the estimated bus voltage at bus $k$, and $\mathrm{V}_{\mathrm{k}}^{\text {ini }}$ is the initial bus voltage at bus $k$ after the initial load flow. $\mathrm{N}_{\mathrm{B}}$ is the number of buses, i.e., 30 .

From Eq. (11) and detailed representation of VSI according to control variables, the estimated voltage can be represented as following equation :

$$
\mathbf{V}_{\mathbf{k}}^{\text {est }}=\mathbf{V}_{\mathbf{k}}^{\text {ini }}+\left[\operatorname{VSI}_{\mathbf{k}}^{\mathrm{C}} \operatorname{VSI}_{\mathbf{k}}^{\mathrm{T}} \operatorname{VSI}_{\mathbf{k}}^{\mathrm{G}}\right] \cdot\left[\begin{array}{c}
\Delta \mathbf{Q}_{\mathrm{k}}^{\mathrm{C}} \\
\Delta \mathbf{Q}_{\mathbf{k}}^{\mathrm{T}} \\
\Delta \mathbf{Q}_{\mathbf{k}}^{\mathrm{G}}
\end{array}\right]
$$

Let us consider the terminal voltage of the DGs as a control variable. The conventional VSI with DG units has significant problem for estimating bus voltage. These errors can ultimately result in an unfeasible solution during the optimization process as shown in Fig. 2.

Because the reactive power produced by DG unit is limited within some specific range, the relationship between terminal voltage and reactive power output of DG unit should be investigated.

The AVSI is introduced to calculate the ratio, $\gamma^{ \pm}$if terminal voltage at the turning point, $\mathrm{V}_{\mathrm{TP}}^{ \pm}$of $\mathrm{DG}$ unit is required. This is computational skill rather than analytical approach. Actually, we modified third component of AVSI row vector in Eq. (13) and defined as Eq. (14).

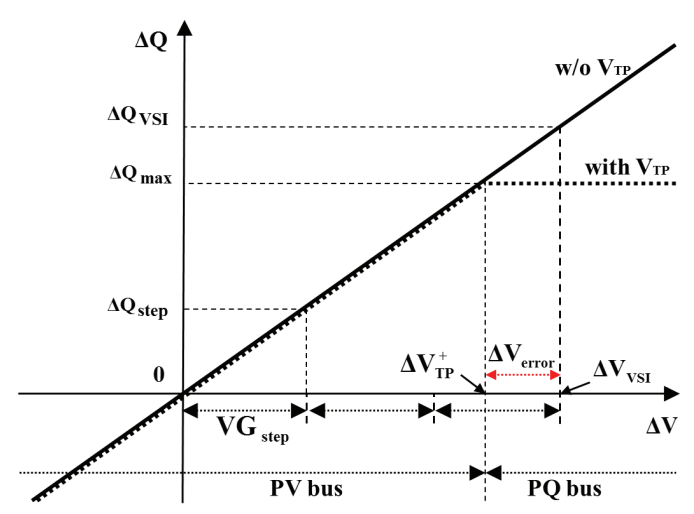

Fig. 2. Advanced voltage sensitivity index considering limited reactive power of DG units 


$$
\operatorname{AVSI}_{k}=\left[\operatorname{VSI}_{\mathbf{k}}^{\mathbf{C}} \operatorname{VSI}_{\mathbf{k}}^{\mathrm{T}} \operatorname{AVSI}_{\mathbf{k}}^{\mathbf{G}}\right]
$$

Such that

$$
\mathbf{A V S I}_{\mathrm{k}}^{\mathrm{G}}=\left\{\begin{array}{cc}
\operatorname{VSI}_{\mathrm{k}}^{\mathrm{G}} & \Delta \mathbf{Q}_{\min } \leq \Delta \mathbf{Q} \leq \Delta \mathbf{Q}_{\max } \\
\infty & \Delta \mathbf{Q}_{\max }<\Delta \mathbf{Q} \| \Delta \mathbf{Q}_{\min }>\Delta \mathbf{Q}
\end{array}\right.
$$

The first row in above equation is VSI of DG unit when DG unit operates PV mode. And the second row means constant $\mathrm{Q}_{\max }$ or $\mathrm{Q}_{\min }$ from $\mathrm{DG}$ unit with $\mathrm{PQ}$ mode. In this operation mode, we can consider the VSI of DG unit is infinity. It means that DG unit is no longer reactive power resource, just constant power load with $\Delta \mathrm{Q}=\Delta \mathrm{Q}_{\max }$ or $\Delta \mathrm{Q}=\Delta \mathrm{Q}_{\min }$ as shown in Fig. 2 .

The upper and lower voltage deviation of the DG are expressed in Eq. (15). It is used to estimate the voltage that is diverted from a PV bus to a PQ bus when the estimated reactive power of the $\mathrm{DG}$ exceeds the minimum or maximum value of the actual reactive power of the DG units.

$$
\begin{aligned}
\Delta \mathbf{V}_{\text {TP }}^{ \pm} & =\left|\frac{\Delta \mathbf{Q}_{\text {max } \| \min }}{\Delta \mathbf{Q}_{\text {step }}}\right| \cdot V_{G_{\text {step }}} \\
& =\left|\frac{\Delta \mathbf{Q}_{\text {max } \| \text { min }}-\mathbf{Q}_{\text {initial }}}{\mathbf{Q}_{\text {initial }}-\mathbf{Q}_{\text {initial }+1}}\right| \cdot \mathbf{V G}_{\text {step }}
\end{aligned}
$$

The ratio, $\gamma^{ \pm}$could be obtained from Eq. (15) and Fig. 2 and used for estimating bust voltage at turning point, $\mathrm{V}_{\mathrm{TP}}^{ \pm}$.

$$
\begin{aligned}
\gamma^{ \pm}=\frac{\Delta \mathbf{V}_{\text {TP }}^{ \pm}-\left[\frac{\Delta \mathbf{Q}_{\text {max } \mid \text { min }}}{\Delta \mathbf{Q}_{\text {step }}}\right] \cdot V_{\text {step }}}{\Delta V S I-\left[\frac{\Delta \mathbf{Q}_{\text {max } \mid \text { min }}}{\Delta \mathbf{Q}_{\text {step }}}\right] \cdot V_{\text {step }}} \\
=\left|\frac{\Delta \mathbf{Q}_{\text {max } \mid \min }}{\Delta \mathbf{Q}_{\text {step }}}\right|-\left[\frac{\Delta \mathbf{Q}_{\text {max } \mid \text { min }}}{\Delta \mathbf{Q}_{\text {step }}}\right]
\end{aligned}
$$

where [] is the square bracket notation for the floor function.

\subsection{Dynamic programming with a heuristic search method}

The optimal dispatch of the control variables can be determined by employing DP [13]. Nevertheless, to determine the optimal control settings, huge search space is required with enormous computational burden [3]. This paper proposes DP along with some techniques for reducing the load-flow iteration number. Proposed approach is applied to a feasible state decision module shown in Fig. 3.

The load-flow calculation modules of existing studies on dynamic programming have been applied for conducting

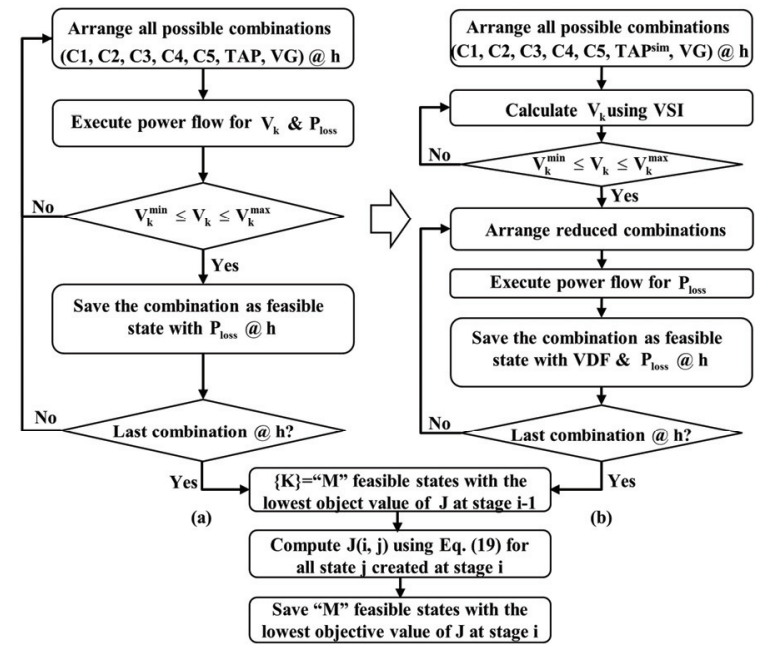

Fig. 3. Feasible state decision module: (a) conventional method and (b) proposed method

the feasibility study to make a decision whether or not to meet the bus voltage bounds and the line capacity, as shown in Fig. 3(a). In these cases, load-flow calculations should be performed regarding entire case at time $i$.

On the other hand, the AVSI described in the previous section of this paper is adopted in order to reduce the number of load-flow calculations by removing the states that violate allowable voltage limits, as shown in the second and third block combination in Fig. 3(b). Consequently, only a reduced set consisting of unviolated states is obtained if voltage sensitivity analysis is conducted prior to load-flow calculation [19]. The estimated bus voltage at bus $k$ can be obtained from VDF table based on VSI matrix and initial bus voltages. It is just required several times based on the number of total control variables for power flow calculation. The computational load in the case of applying the AVSI to the load-flow calculation process includes the voltage sensitivity analysis and the active power loss calculation. It could be represented as following equation:

$$
\mathbf{0} \leq \mathbf{N}_{\mathrm{VSI}}+\boldsymbol{\alpha}_{\mathbf{o b s}, \mathbf{i}} \cdot \mathbf{L}_{\mathbf{i}}^{\max } \leq \mathbf{L}_{\mathbf{i}}^{\max }
$$

where $\alpha_{\text {obs,i }}$ is defined as the observation rate that satisfies the voltage limit at time $i, \mathrm{~N}_{\mathrm{VSI}}$ is the total number of all controllers, and $\mathrm{L}_{\mathrm{i}}^{\max }$ is the maximum number of controls at each stage $i$. The size of the reduced set depends on the time $i$ and the allowed voltage limits.

The accumulated switching operation number up to the current stage is applied prior to the load-flow calculation module, as shown in Fig. 3(b). It can remove the states that exceed the maximum switching operation number. The computational burden has greatly reduced by using DP with a heuristic search method.

In addition to the AVSI and the SON, the limited operating range of OLTCs needs to be considered.

Unless DG units are not included as a control variable, 
the tap operating range could be limited by estimating the ideal tap position using a mathematical model of the OLTCs, shunt capacitor, and load at time $i$. However, because the presence of DGs necessitates a more complicated calculation process, it is difficult to apply conventional estimation schemes for determining an ideal tap position in this study. In this work, simplified DP (SDP) in [10-11] is adopted for determining an appropriate setting value of the tap position referred to the secondary reference voltage of the OLTCs.

The size of the reduced combination set at each stage is represented by the following equation:

$$
\mathbf{L}_{\mathbf{i}}=\mathbf{N}_{\text {BASE }}+\boldsymbol{\alpha}_{\text {obs,i }} \cdot \boldsymbol{\beta}_{\text {sim }} \cdot \mathbf{L}_{\mathbf{i}}^{\max }-\mathbf{N}_{\text {SON }}
$$

where $\beta_{\text {sim }}$ is the rate of with and without SDP with a specific range of OLTC tap positions [10].

Generally, the recursive equation for applying the DP is represented as

$$
\mathbf{J}(\mathbf{i}, \mathbf{j})=\min _{\{\mathbf{K}\}}\left[\mathbf{J}(\mathbf{i}-\mathbf{1}, \mathbf{K})-\mathbf{J}_{\mathbf{c}}(\mathbf{i}, \mathbf{j})\right]
$$

where $J(i, j)$ is defined as the minimum accumulated objective function up to $\operatorname{stage}(i, j)$ and $J_{c}(i, j)$ is the objective function for stage $(i, j)$.

\subsection{Computational procedure}

The five interconnected modules shown in Fig. 4 were designed and implemented based on PSS/E with python to determine the optimal solution of the objective function

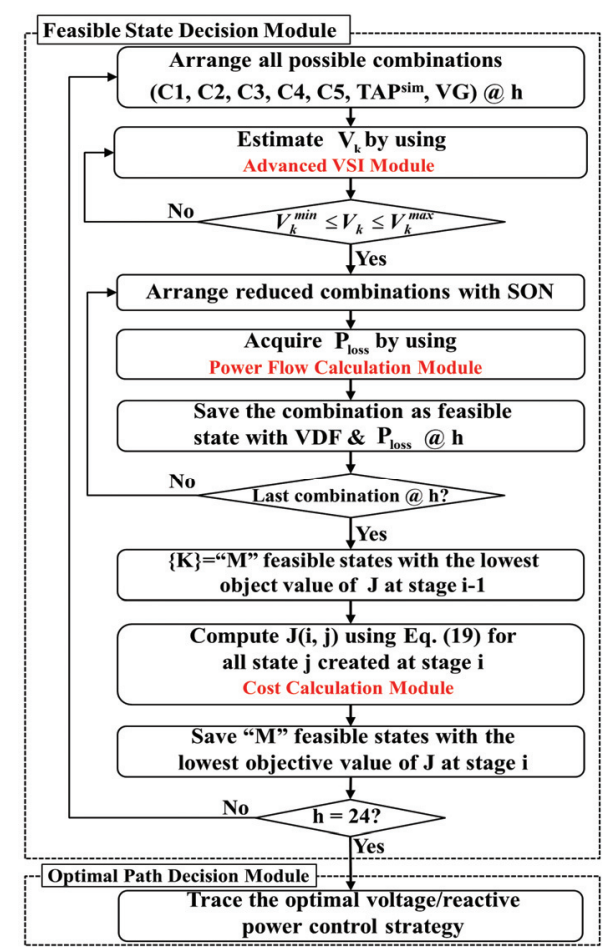

Fig. 4. Methodology of optimal scheduling for ORPD 4. Case Study under the constraint conditions using the proposed approach. The python script was used to develop the proposed approach with the API of PSS/E and run automatically. The feasible state decision process is iterated until the final stage is reached. The optimal voltage and reactive power schedules are obtained by using the optimal path decision module including the backtracking algorithm.

\section{Case Study}

\subsection{System configuration}

The IEEE 30-bus test system shown in Fig. 5 was employed to verify the proposed control objective and evaluate the solution algorithm [20].

Table 1 shows a summary of the control variables including OLTC, DG unit, and fixed shunt capacitors. And the load model for the power-flow studies was considered as constant power [13].

\subsection{Daily load and generation variations}

Assuming that the network is scanned every one hour, the network is scanned 24 times. In Fig. 6, the load level changes from $45 \%$ to $100 \%$ for peak load condition with $88 \%$ lagging power factor. The DG output power varies from $74 \%$ to $100 \%$ for the rated capacity, which is $3 \mathrm{MW}$.

\subsection{Simulation results}

The estimated bus voltage magnitudes should be enough to be similar to the actual values obtained from the newtonraphson load flow (NRLF) module of PSS/E.

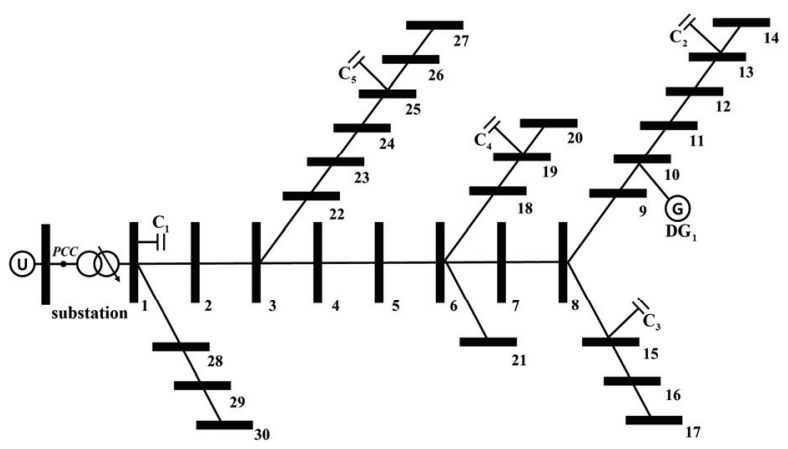

Fig. 5. IEEE 30-bus test system

Table 1. Definition of control variable

\begin{tabular}{c|c|c}
\hline Variable & Type & Range (p.u.) \\
\hline C1 & Integer & {$[0,1]$} \\
\hline C2 & Integer & {$[0,1]$} \\
\hline C3 & Integer & {$[0,1]$} \\
\hline C4 & Integer & {$[0,1]$} \\
\hline C5 & Integer & {$[0,1]$} \\
\hline OLTC1 & Discrete & {$[0.90,0.91, \ldots, 1.09,1.10]$} \\
\hline DG1 & Discrete & {$[0.95,0.96, \ldots, 1.04,1.05]$} \\
\hline
\end{tabular}




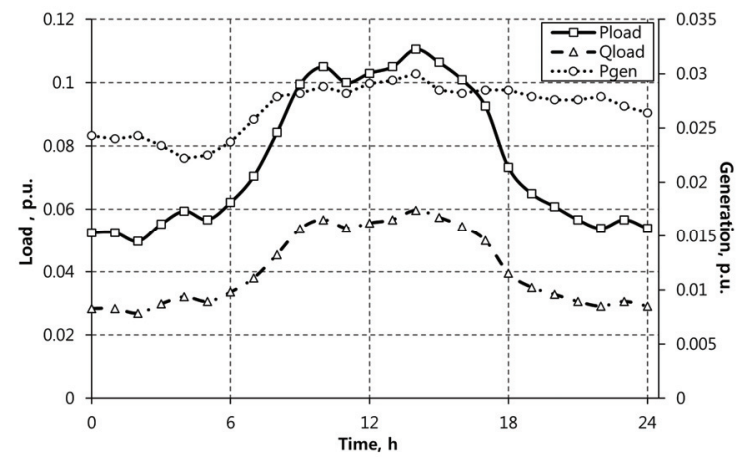

Fig. 6. Daily load demand and active power of DG output

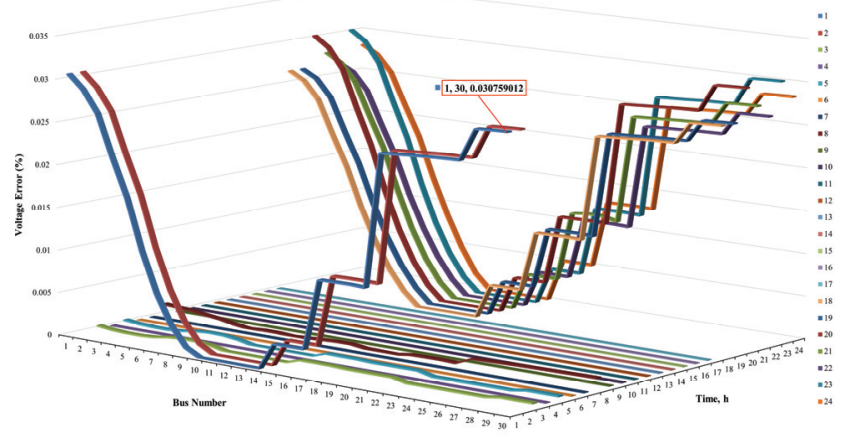

Fig. 7. Percent errors in bus voltage estimation for all stages as a function of bus number and time

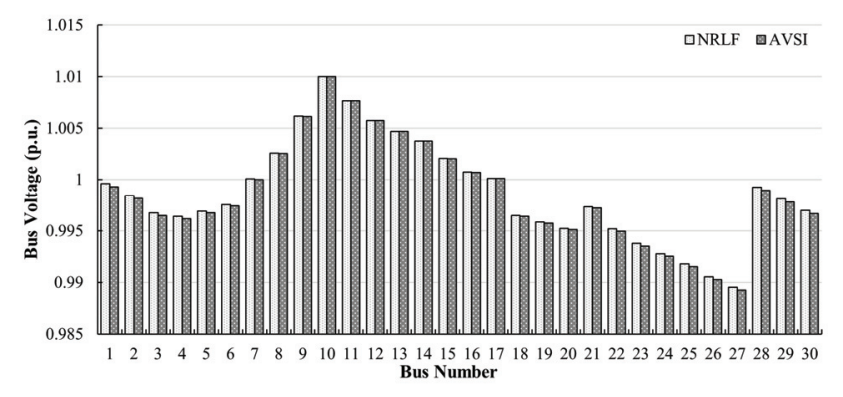

Fig. 8. Comparison results of bus voltage magnitudes obtained from NRLF and AVSI

$$
V_{\text {error,k }}=\frac{\left|V_{k}^{\text {est }}-V_{k}^{\text {act }}\right|}{V_{k}^{\text {act }}} \cdot 100[\%]
$$

The percent errors using Eq. (20) of bus voltage estimation for all stages are plotted as a function of bus number and time shown in Fig. 7.

At 01:00, we can find out that the maximum voltage error is $0.030759 \%$ at node 30 . Even in this case, the estimated bus voltage magnitudes are significantly similar to actual values shown in Fig. 8 .

The second results are concerned with the computation time for different cases. This paper proposes the use of the DP algorithm to overcome the computational burden of the iterative power flow calculation. The parameters, including the daily maximum SON of each controller, length of the
Table 2. Parameters used in the proposed method

\begin{tabular}{c|c|c|c|c|c}
\hline \multirow{2}{*}{$\mathrm{M}$} & \multicolumn{3}{|c|}{ Maximum Switching Operation } & \multicolumn{2}{c}{ Cost-based Factors } \\
\cline { 2 - 6 } & $\mathrm{MK}_{\mathrm{T}}$ & $\mathrm{MK}_{\mathrm{C}}$ & $\mathrm{MK}_{\mathrm{G}}$ & $\mathrm{C}_{\mathrm{P}_{\text {loss }}}$ & $\mathrm{C}_{\mathrm{SC}}$ \\
\hline 150 & 30 & 10 & 2 & 65 & 106,944 \\
\hline
\end{tabular}

Table 3. Comparison with computation time for applied methods

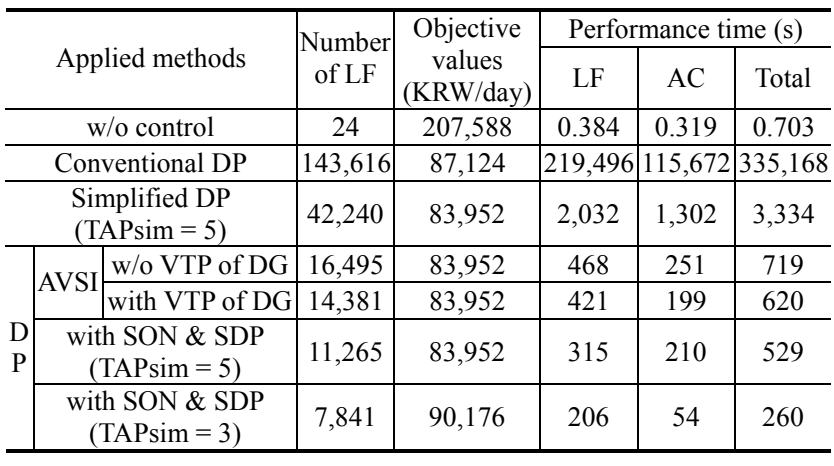

search space at each stage, and cost factor, are summed up in Table 2.

The comparison results for before control, conventional DP, SDP, and proposed approach including load-flow calculation process and arrange combinations process are summarized in Table 3. Conventional DP should perform the load flow for all search spaces of each controller. Even if this method ensures that it always provides the global solution, it is difficult to apply to real power systems owing to its impractical computation time. Therefore, SDP with appropriate range of TAP $\left(\mathrm{TAP}_{\text {sim }}=5\right)$ was selected as the basis of this comparison analysis [10]. And the reduced number of load flow for proposed approach are dependent on effectiveness of applied methods. As shown in Table 3, the use of proposed approach reduced the performance time by at least $92 \%$ compared with SDP as the base method.

The total annual electricity charges and benefits of the proposed method with 3 cases are summarized in Table 4. The total real power losses, power factors, and their costbased values are also represented in Table 4.

Generally, an improved receiving power factor should result in reduced active power losses. However, comparing loss minimization with the proposed method could indicate that receiving power and active power losses are not directly related. Using the loss minimization approach, the active power loss should be minimized. When our control objective function for minimizing the electric charge is applied to optimal scheduling strategy, we try to minimize daily total electricity charges rather than active power loss. Although our proposed approach is relatively unfavorable in terms of active power loss, we assure that total electricity charges can be minimized by improving the receiving power factor, as shown in Table 4.

In terms of system losses, the proposed method can 
Table 4. Comparison of simulation results

\begin{tabular}{c|c|c|c}
\hline & $\begin{array}{c}\text { w/o } \\
\text { control }\end{array}$ & $\begin{array}{c}\text { Loss } \\
\text { minimization }\end{array}$ & $\begin{array}{c}\text { Proposed } \\
\text { method }\end{array}$ \\
\hline $\begin{array}{c}\text { Daily active power } \\
\text { losses (kW) }\end{array}$ & $3,078.5$ & $2,362.1$ & $2,374.5$ \\
\hline Power factor (\%) & 89.45 & 96.68 & 97.09 \\
\hline VDF $_{\text {imp }}(\%)$ & 0 & 91.42 & 91.87 \\
\hline $\begin{array}{c}\text { Daily cost of active } \\
\text { power losses (won) }\end{array}$ & 200,102 & 153,534 & 154,343 \\
\hline Daily cost of PF (won) & 7,486 & $-58,284$ & $-64,166$ \\
\hline $\begin{array}{c}\text { Annual electric charges } \\
\text { (AEC) (million won) }\end{array}$ & 75.7696 & 34.7660 & 32.9143 \\
\hline Annual saving (\%) & 0 & 54.12 & 56.56 \\
\hline
\end{tabular}

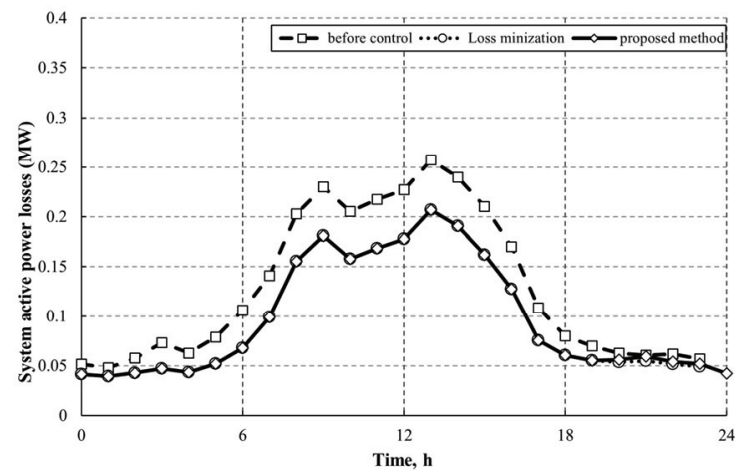

Fig. 9. Comparison of active losses for an entire day

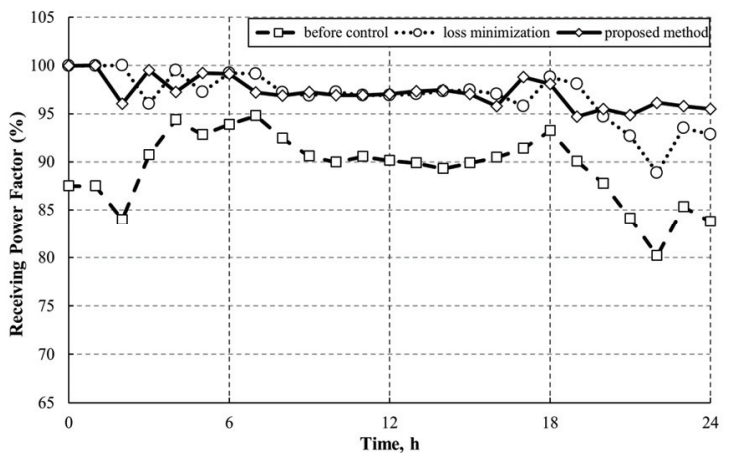

Fig. 10. Receiving power factors for an entire day

reduce the power consumed by $22.9 \%$. Loss minimization can save the $12.4 \mathrm{~kW}$ fewer power losses than the proposed method. However, the average receiving power factor after using the proposed method is improved to $97.09 \%$ as compared with $89.45 \%$ before control and $96.68 \%$ with loss minimization. In terms of annual benefits, the saving of electricity charges using the proposed method is $2.44 \%$ more than that using loss minimization. System active power losses for an entire day are represented in Fig. 9.

The receiving power factors at each hour for the three different cases are shown in Fig. 10. The proposed method attempts to maintain the receiving power factor within the maximum incentive margin, i.e., $95 \%$ from 00:00 to 24:00. This plot indicates that the proposed method performs satisfactorily in maintaining the receiving power factor at the desired value.
Table 5. Optimal control strategy for loss minimization (A) and electricity charges savings (B)

\begin{tabular}{|c|c|c|c|c|c|c|c|c|c|c|c|c|c|c|}
\hline \multirow{2}{*}{ hour } & \multicolumn{2}{|c|}{$\mathrm{C} 1$} & \multicolumn{2}{|c|}{$\mathrm{C} 2$} & \multicolumn{2}{|c|}{$\mathrm{C} 3$} & \multicolumn{2}{|c|}{$\mathrm{C} 4$} & \multicolumn{2}{|c|}{$\mathrm{C} 5$} & \multicolumn{2}{|c|}{ TAP1 } & \multicolumn{2}{|c|}{ VG1 } \\
\hline & $\mathrm{A}$ & $\mathrm{B}$ & $\mathrm{A}$ & $\mathrm{B}$ & $\mathrm{A}$ & $\mathrm{B}$ & $\mathrm{A}$ & $\mathrm{B}$ & $\mathrm{A}$ & $\mathrm{B}$ & $\mathrm{A}$ & $\mathrm{B}$ & $\mathrm{A}$ & $\mathrm{B}$ \\
\hline 1 & 1 & 1 & 1 & 1 & 1 & 1 & 1 & 1 & 1 & 1 & 0 & 0 & 1.01 & 1.01 \\
\hline 2 & 1 & 1 & 1 & 1 & 1 & 1 & 1 & 1 & 1 & 1 & 0 & 0 & 1.01 & 1.01 \\
\hline 3 & 0 & 0 & 1 & 1 & 1 & 1 & 1 & 1 & 1 & 1 & -1 & -1 & 1.01 & 1.01 \\
\hline 4 & 1 & 1 & 1 & 1 & 1 & 1 & 1 & 1 & 1 & 1 & -1 & -1 & 1.01 & 1.01 \\
\hline 5 & 0 & 0 & 1 & 1 & 1 & 1 & 1 & 1 & 1 & 1 & -1 & -1 & 1.01 & 1.01 \\
\hline 6 & $\begin{array}{ll}1 \\
\end{array}$ & 1 & 1 & \begin{tabular}{l|l|}
1 \\
\end{tabular} & 1 & 1 & 1 & \begin{tabular}{l|l|}
1 \\
\end{tabular} & 1 & 1 & -1 & -1 & 1.01 & \begin{tabular}{|l|}
1.01 \\
\end{tabular} \\
\hline 7 & 1 & 1 & 1 & 1 & 1 & 1 & 1 & 1 & 1 & 1 & -1 & -1 & 1.01 & 1.01 \\
\hline 8 & 1 & 1 & 1 & 1 & 1 & 1 & 1 & 1 & 1 & 1 & -2 & -2 & 1.01 & $\begin{array}{ll}1.01 \\
\end{array}$ \\
\hline 9 & 1 & 1 & 1 & 1 & 1 & 1 & 1 & 1 & 1 & 1 & -2 & -2 & 1.00 & 1.00 \\
\hline 10 & 1 & 1 & 1 & 1 & 1 & 1 & 1 & 1 & 1 & 1 & -2 & -2 & 1.00 & 1.00 \\
\hline 11 & 1 & 1 & 1 & 1 & 1 & 1 & 1 & 1 & 1 & 1 & -2 & -2 & 1.00 & 1.00 \\
\hline 12 & 1 & 1 & 1 & 1 & 1 & 1 & 1 & 1 & 1 & 1 & -2 & -2 & 1.00 & 1.00 \\
\hline 13 & 1 & 1 & 1 & 1 & 1 & 1 & 1 & 1 & 1 & 1 & -2 & -2 & 1.00 & 1.00 \\
\hline 14 & 1 & 1 & 1 & 1 & 1 & 1 & 1 & 1 & 1 & 1 & -2 & -2 & 1.00 & 1.00 \\
\hline 15 & 1 & 1 & 1 & \begin{tabular}{l|l|}
1 \\
\end{tabular} & 1 & 1 & 1 & 1 & 1 & 1 & -2 & -2 & 1.00 & 1.00 \\
\hline 16 & 1 & 1 & 1 & 1 & 1 & 1 & 1 & 1 & 1 & 1 & -2 & -2 & 1.00 & 1.00 \\
\hline 17 & 1 & 1 & 1 & 1 & 1 & 1 & 1 & 1 & 1 & 1 & -2 & -2 & 1.00 & 1.00 \\
\hline 18 & 1 & 1 & 1 & 1 & 1 & 1 & 1 & 1 & 1 & 1 & 0 & 0 & 1.00 & 1.00 \\
\hline 19 & 1 & 1 & 1 & 1 & 1 & 1 & 1 & 1 & 1 & 1 & 0 & 0 & 1.00 & 1.00 \\
\hline 20 & 0 & 0 & 1 & 1 & 1 & 1 & 1 & 1 & 1 & 1 & 0 & 0 & 1.00 & 1.00 \\
\hline 21 & 0 & 1 & 1 & 1 & 1 & 1 & 1 & 0 & 1 & 1 & 0 & 0 & 1.00 & 1.00 \\
\hline 22 & 0 & 1 & 1 & 1 & 0 & 1 & 1 & 1 & 1 & 1 & 0 & 0 & 1.00 & 1.00 \\
\hline 23 & 0 & 1 & 1 & 1 & 1 & 0 & 1 & 1 & 1 & 1 & 0 & 0 & 1.00 & 1.00 \\
\hline 24 & 0 & 1 & 1 & \begin{tabular}{l|l|}
1 \\
\end{tabular} & 1 & 0 & 1 & 1 & 1 & 1 & 0 & 0 & 1.00 & 1.00 \\
\hline SON & 5 & 6 & 0 & 0 & 2 & 1 & 0 & 2 & 0 & 0 & 5 & 5 & 2 & 2 \\
\hline
\end{tabular}

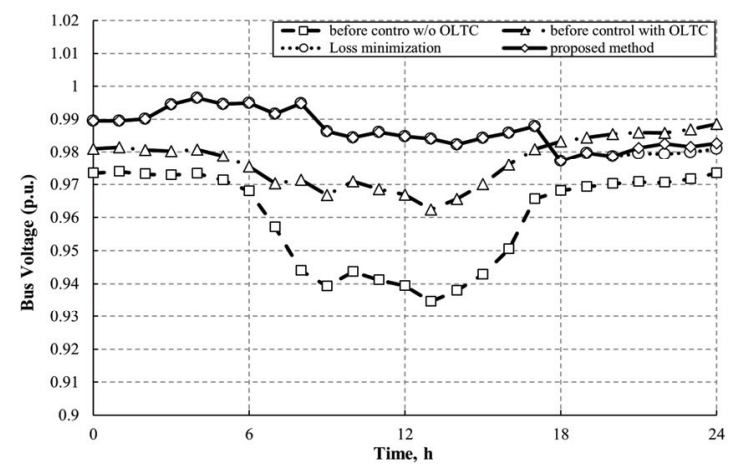

Fig. 11. Comparison of daily voltage profile at node 27

Table 5 shows the optimal dispatch results of the shunt capacitors, DG unknit, and OLTC. The initial tap position of the OLTC is -2 , the initial set point of the DG unit is 1.00 p.u., and the original status of all capacitors $(\mathrm{C} 1 \sim \mathrm{C} 5)$ is on, i.e., 1 .

The number of switching operations for the OLTC for the entire day is five. SON for the DG unit is two. $\mathrm{C} 1$ changes six times in a day, $\mathrm{C} 2$ and $\mathrm{C} 5$ are on for the entire day, C3 switches only once, and C2 changes two times.

Fig. 11 shows the voltage profiles at node 27 for four different cases since the node 27 is a weak point with the lowest voltage magnitude in the test system. However, in Fig. 11, the bus voltages at node 27 before control exceed the allowed voltage limits, i.e., 0.97 p.u., even if the OLTC is operated with AVR. In this case, with and without the 


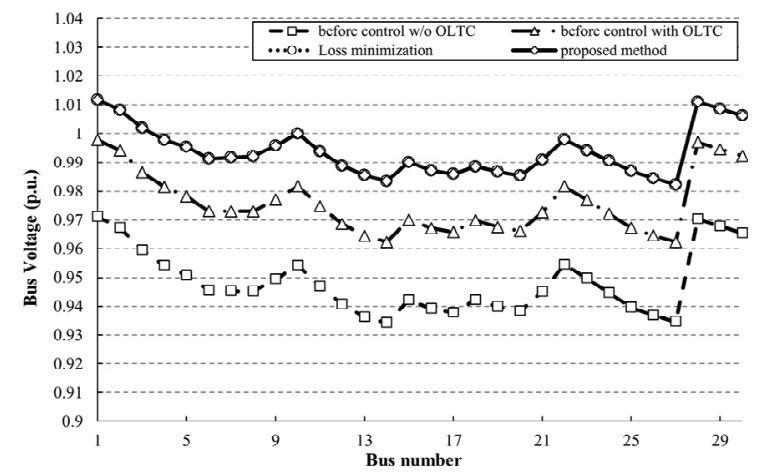

Fig. 12. Voltage profiles at peak time

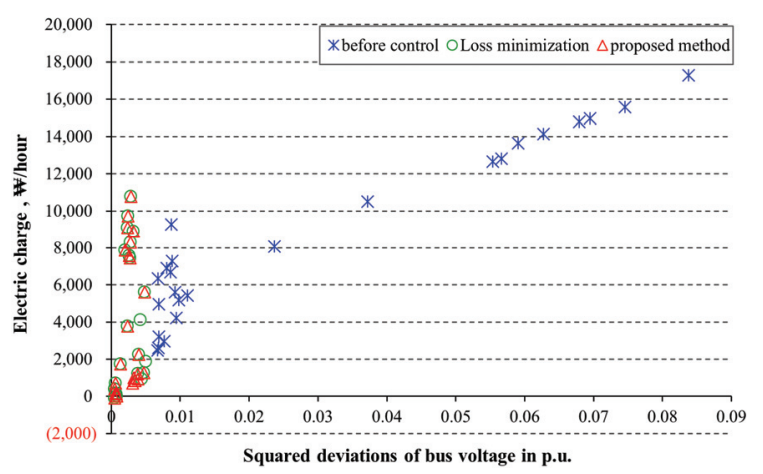

Fig. 13. Scatter plot between VDF and electric charges

OLTC, the minimum bus voltages are 0.935 p.u. and 0.962 p.u., respectively.

These results show a tendency to trace the daily load demand and the DG output, which is shown in Fig. 6. On the contrary, all the bus voltages of the entire test system after loss minimization and the proposed method are maintained within the allowable voltage ranges. The voltage profile at 14:00 when the test system was subjected to a heavy load is greatly improved. Fig. 12 shows the voltage profiles at 14:00 for the four different cases.

Fig. 13 shows the correlation between the VDF and the total electrical charges using scatter plots. From Fig. 13, we make the following observations. First, the total electrical charges and the VDF are relatively reduced. Second, the scatter plot of the proposed method show that both the losses and the VDF are greatly reduced than in other cases.

The feasible states with the lowest objective value by calculating the control objective with several constraints could be saved at each stage $i$. These are less than those observed in simulation results of before control and loss minimization. This means that the proposed method is more effective in terms of saving the electric charges and improving the voltage profile than loss minimization.

\section{Conclusion}

A novel optimal voltage control and reactive power dispatch schedule is proposed in this study. The control variables include the status of shunt capacitor switching, tap position of OLTCs, and voltage set point of DGs. The combined objective function, including the receiving power factor and loss minimization, minimized the electric charges and determined the optimum dispatch schedules. The proposed method was implemented using a python script and the results were applied for PSS/E. Optimal dispatch schedules were obtained by using proposed approach with a VSI and other techniques. To avoid the computational burden, an AVSI was adopted to reduce the number of load-flow calculations by estimating bus voltages. Moreover, the accumulated switching operation number up to the current stage was applied prior to the load-flow calculation module. This step can remove the states that exceed the maximum switching operation number.

The proposed algorithm was applied to the IEEE 30bus test system. The results obtained through these case studies are as follows. First, proposed approach can reduce the performance time by at least $92 \%$ as compared with SDP. Second, the results obtained using the proposed method were compared with simulation results obtained before control and from an algorithm that only considered loss minimization. The results indicated that the proposed method is more effective in terms of saving the electric charges and improving the voltage profile than loss minimization.

In conclusion, it is anticipated that the proposed algorithm described in this paper will contribute to the development of an intelligent energy management system with automation. The ORPD problem has also been studied using metaheuristic methods such as GA, SA, and TS. Nevertheless, it is difficult to directly compare our results with their results because of the differences of the control objective function, type and number of control variables, size of the test system, and uncertain simulation results of existing studies. Hence, we have compared our simulation results with the results of existing dynamic programming approaches. In terms of execution time, the time-interval of voltage/var dispatch function of EMS in korea power exchange is set to 4 minutes to automatically run.

In the future, advanced load forecasting techniques and PSS/E automation techniques need to be studied and applied for the development of automatic real-time control systems with feasible time intervals. In addition, we will focus on applying the advanced solution engine and more sophisticated sensitivity analysis with over two DGs for finding the feasible solution based on approximate linear relationship.

\section{Acknowledgements}

This research was supported by a grant (15RTRPB067917-03) from the Railroad Technology Research Program funded by the Ministry of Land, Infrastructure, and Transport, Republic of Korea. 


\section{References}

[1] K. R. C. Mamandur, R. D. Chenoweth, "Optimal Control of Reactive Power Flow for Improvements in Voltage Profiles and for Real Power Loss Minimization," IEEE Trans. Power App. Syst., vol. PAS-100, no. 7, pp. 3185-3194, 1981.

[2] P. Ekel, S. Ansuj, R. Schinzinger, A. Prakhovnik, O. Razumovsky, "Automation of Reactive Power Compensation in Industrial Power Systems," Proc. of the Third IEEE Conf. on Control App., Glasgow, UK, Aug. 1994.

[3] Z. Hu, X. Wang, H. Chen, and G. A. Taylor, "Volt / VAr control in distribution systems using a timeinterval based approach," IEE Proceedings-Generation, Transmission and Distribution, vol. 150, no. 5, pp. 548-554, 2003.

[4] Mohd Zamri Che Wanik, Istvan Erlich, and Azah Mohamed, "Intelligent Management of Distributed Generators Reactive Power for Loss Minimization and Voltage Control," IEEE MELECON, Valletta, Malta, April 2010.

[5] J. O'Donnel, Voltage management of networks with distributed generation, Ph.D. dissertation, Univ. of Edinburgh, 2007.

[6] Duong Quoc Hung, Nadarajah Mithulananthan, "Multiple Distributed Generator Placement in Primary Distribution Networks for Loss Reduction," IEEE Trans. on Ind. Elect., vol. 60, no. 4, pp. 1700-1708, 2013.

[7] Wen Zhang, Yutian Liu and Yuanqi Liu, "Optimal Var Planning in Area Power System," Proc. PowerCon 2002, Chengdu, China, Oct. 2002.

[8] Hsiao YT, Chiang HD, Liu CC, Chen YL, "A computer package for optimal multi-objective VAR planning in large scale power system," IEEE Trans. Power Syst., vol. 9, no. 2, pp. 668-676, 1994.

[9] M. E. Hamedani Golshan and S. A. Arefifar, "Distributed generation, reactive sources and networkconfiguration planning for power and energy-loss reduction," IEE Proceeding- Generation, Transmission and Distribution, vol. 153, no. 2, pp. 127-136, 2006.

[10] Lu, F.-C., and Hsu, Y.-Y., "Reactive power/voltage control in a distribution substation using dynamic programming," IEE Proceeding-Generation, Transmission and Distribution, vol. 142, no. 6, pp. 639-645, 1995.

[11] Ruey-Hsun Liang, Chen-Kuo Cheng, "Dispatch of main transformer ULTC and capacitors in a distribution system," IEEE Trans. Power Del., vol. 16, no. 4, pp. 625-630, 2001.

[12] Young-Jin Kim, Seon-Ju Ahn, Pyeong-Ik Hwang, Gi-Chan Pyo and Seung-Il Moon, "Coordinated Control of a DG and Voltage Control Devices Using a Dynamic Programming Algorithm," IEEE
Trans. Power Syst., vol. 28, no. 1, pp. 42-51, 2013.

[13] K. S. Jeong, H. C. Lee, Y. S. Baek and J. H. Park, "Coordinated Voltage and Reactive Power Control Strategy with Distributed Generator for Improving the Operational Efficiency," J. Elect. Eng. Technol., vol. 8, no. 6, pp. 1261-1268, 2013.

[14] Vovos, P.N., Kiprakis, A.E., Wallace, A.R., Harrison, G.P., "Centralized and Distributed Voltage Control: Impact on Distributed Generation Penetration," IEEE Trans. Power Syst., vol. 22, no. 1, pp. 476-483, 2007.

[15] Siemens PTI, PSS/E-32 Program Application Guide, Siemens Power Technologies International Inc., 2009.

[16] Ki Seok Jeong, Hyun Chul Lee, Ji Ho Park and Young Sik Baek, "Optimal dispatch scheduling with synchronous machines for improving operational efficiency in industrial power system," IEEE ICSIMA, Kuala Lumpur, Malaysia, Nov. 2013.

[17] P. Kundur, Power System Stability and Control, McGraw-Hill Inc., 1994.

[18] Seshadri, P.S., Patton, A.D., "Bus Voltage Sensitivity: An Instrument for Pricing Voltage Control Service," IEEE Power Engineering Society Summer Meeting, Edmonton, Canada, July 1999.

[19] Ki-Seok Jeong, Hyun-Chul Lee, Ji-Ho Park and Young-Sik Baek, "Voltage and Reactive Power Coordination Control for Improving Voltage Profile Using VQ Sensitivity in power system with Renewable Energy Sources," International Smart Grid Conference \& Exhibition 2013, Jeju, Republic of Korea, July 2013.

[20] S. Civanlar, J. J. Grainger, "Volt/Var Control on Distribution Systems with Lateral Branches Using Shunt Capacitors and Voltage Regulators Part III: the Numerical Results," IEEE Trans. Power App. Syst., vol. PAS-104, no. 11, pp. 3291-3297, 1985.

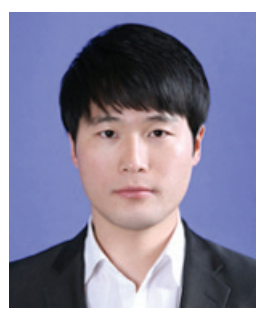

Ki-Seok Jeong He received his B.S., M.S., and Ph.D. degrees in Electrical Engineering from Kyungpook National University, Korea, in 2008, 2010 and 2014 , respectively. He is currently a postdoctoral researcher in the Metropolitan Transit Convergence Research Division at Korea Railroad Research

Institute (KRRI).

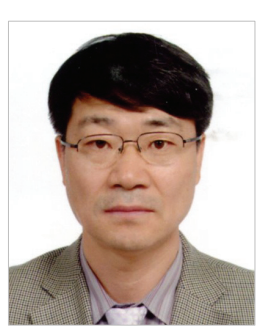

Jong-Duk Chung He received his $\mathrm{Ph} . \mathrm{D}$. degree in Precision Mechanical Engineering from Hanyang University, Korea, in 2006 . He is currently a chief researcher in the Metropolitan Transit Convergence Research Division at Korea Railroad Research Institute (KRRI). 